\title{
Kurzgeschichte der Placentarforschung
}

\author{
Von Fritz Strauss
}

\section{Einleitung}

Da kein Mammalier ohne intrauterine Verbindung zum foeto-maternen Austauschorgan zur Welt kommen kann, mag es angezeigt erscheinen, im Rahmen des noch vor 200 Jahren wenig klaren Entwicklungsweges der Placenta den Versuch zu wagen, in einem Überblick die in den letzten Jahrhunderten geäußerten Ansichten über die Bildung des Frucht- oder Mutterkuchens und seiner Aufgabe sowie die seiner Bedeutung wegen erforderlichen Vorbereitungen zu verfolgen.

Dabei wäre es verlockend, die im Altertum und Mittelalter über die Placenta vertretenen Auffassungen, die sich in Mythen und Sagen wie in der Heiligen Schrift (5. Buch Mose, Deuteronomium 28: 57; 1. Buch Samuel, 25) widerspiegeln, zu berücksichtigen. Infolge des in jenen Zeiten gebotenen überreichen Stoffes ist es hier nicht möglich, auf die entsprechenden Originalveröffentlichungen einzutreten.

\section{Uterus}

Bei einer medicinhistorischen Betrachtung des placentaren Geschehens und seiner Vorbereitungen ist es des Verständnisses der differenten Begriffe wegen wesentlich, einen kurzen Blick auf den Uterus, quasi auf das Biotop der Placenta, zu werfen.

Wenn man außer der tierischen auch die menschliche Gebärmutter Uterus nennt, ist man gegenüber den Damen unhöflich, denn Uterus kommt von Uter, dem Schlauch (Hyrtl 1880). Das ist der menschliche Fruchtträger nicht. Der Vergleich mit Schlauch geht auf die mächtigen Uterusschläuche trächtiger Haustiere zurück, weshalb Vesal (1514-1564), der als erster die Fachbezeichnungen Uterus und Cervix gebrauchte, Galen (129-199) vorwarf, er habe seine bei Tiersectionen erhobenen Feststellungen ohne weiteres auf den Menschen übertragen (Faller 1960). Weil die Mehrzahl der secierten Säugetiere schon im Altertum vorwiegend Ungulaten mit Uterus duplex bzw. bicornuatus waren, lag es nahe, sich den menschlichen, nichtschwange- 
ren Fruchtträger, der piriform ist, jenen gleichgestaltet vorzustellen. Deshalb überrascht es nicht. lal.3 rom Frühmittelalter an lange mit Nachdruck

\section{figuratuattitis}

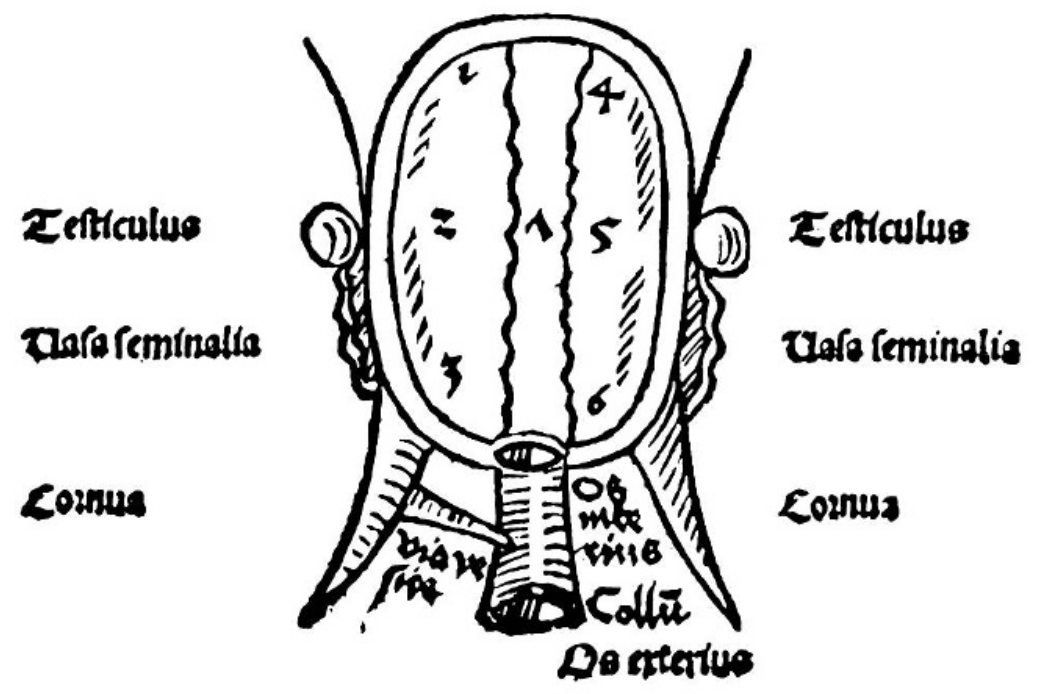

Abb. 1 demonstriert die etwa zu Beginn des 16. Jahrhunderts herrschende und zeichnerische von Hundt (1501) vertretene Vorstellung über den Bau der menschlichen Gebärmutter

(aus Ramsey 1973)

die Annahme vertreten wurde, die menschliche Gebärmutter, die Matrix (= Uterus), bestehe aus 7 Hohlräumen, von denen sich je 3 links bzw. rechts befänden (Abb.1). Dazu wurde angenommen, die männlichen Früchte würden sich in der rechten (der Nähe der Leber wegen) und die weiblichen in der linken Kammer entwickeln; die Hermaphroditen sollten sich in der zentralen Kammer ausbilden (Ramsey 1973). Gewisse morphologische Fortschritte, die wir der Renaissance verdanken, können wir im Vergleich zu Dürer, Michelangelo, Raphael u.a. vor allem Leonardo Da Vinci (14521519) zuschreiben, der einen wesentlichen Teil seiner Interessen in der Anatomie sah. Er brach mit der traditionellen Vorstellung des bicornuaten Uterus und beschrieb die menschliche Gebärmutter als aus nur einer Cavität bestehend. Der tatsächlichen Situation des Fruchthalterbaues nähern sich deshalb die Zeichnungen von Leonardo. Eine klare und der Form des Uterus humanum sehr nahe kommende Vorstellung gab zu Beginn des 16. Jahr- 
hunderts auch Hundt (1501) bildhaft wieder (Ramsey 1982) (Abb.2). Die hier genannten Forscher und Künstler beschäftigten sich praktisch mit der

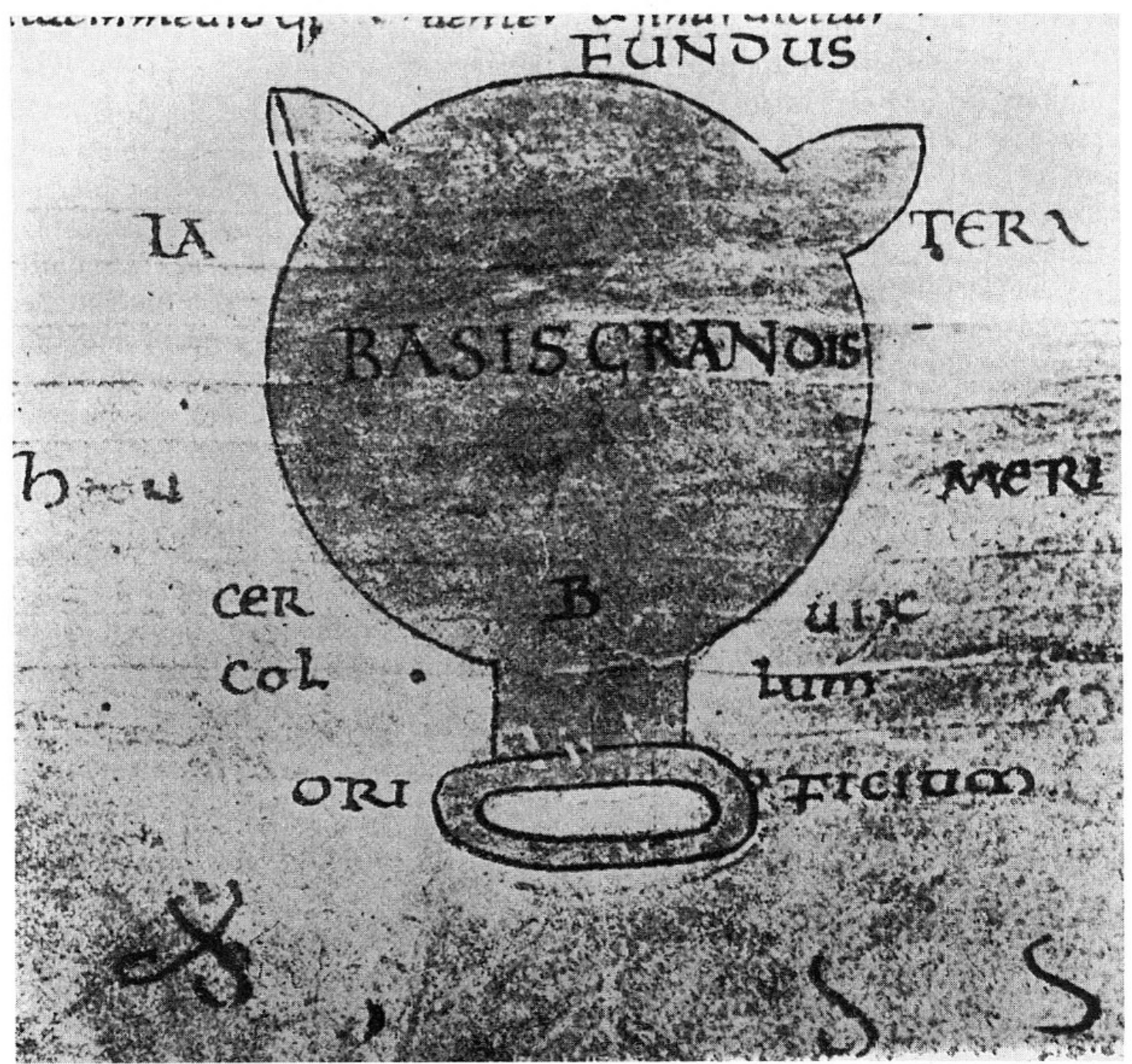

Abb. 2 zeigt den Uterus humanus nach Auffassung von Soranus (98-138 n. Chr.)

(aus Ramsey 1973)

uterinen Morphologie, während William Harvey (1578-1657), der Kreislaufforscher, auch das Problem der Conception in seinem Werk «De generatione animalium» erörterte. Aufgrund unseres heutigen Wissens fand er verständlicherweise die Gebärmutter post coitum stets leer, was ihm verschiedene heftige Angriffe einbrachte (Goltz 1986). 


\section{Menstruation}

Obwohl die Monatsblutung bereits im Alten Testament erwähnt ist, war nicht bekannt, daß sie eine Ausscheidung der Gebärmutter ist. Jedoch schon Mose hat sich in seinem 3. Buch (15: 19-23, 25 u. 33) mit der Periodenblutung befaßt und sehr ausführlich «Von leiblicher Unsauberkeit» gesprochen, so daß vermutet werden kann, ihm seien die Beziehungen zwischen Uterus und monatlicher Blutung bekannt gewesen. Trotz der mosaischen Ausführungen wurde während vieler Jahrhunderte die Regelblutung aufgrund der unbekannten physiologischen Zusammenhänge als ein geheimnisvoller Vorgang betrachtet, der bis gegen Ende des vergangenen Jahrhunderts verschiedene Theorien auslöste, über die unter unterschiedlichen Perspektiven FischerHomberger (1979) und Müller-Hess (1938) orientieren. Mit Einsatz der verbesserten Lebensbedingungen während des 18 . Jahrhunderts nahm man mit Jean-Jaques Rousseau (1712-1778) an, die Regelblutung sei die Folge eines allzu üppigen Lebenstils; damals wurde auch die Ansicht geäußert, sie sei ein widernatürlicher Vorgang (Müller-Hess). Gegen Ende des 18. Jahrhunderts führte der Nosologe W. Cullen (1710-1790), Professor der Medicin in Edinburgh, die Auslösung der Menses auf einen Reiz eines in den Eiern eingeschlossenen Saftes zurück; diesem schrieb er die Aufgabe zu, den Uterus auf die Keimaufnahme vorzubereiten und den Keim in den ersten Monaten zu ernähren (Plaut 1969). Oken (1805) sah in der Menstruation eine Folge «der fortdauernden Eingeschränktheit des Geschlechtstriebes», sie «erbe sich von der Mutter auf die Tochter fort». Etwa gleichzeitig äußerte R. Remak (1815-1865) (Fischer-Homberger) die Ansicht, die Regelblutung sei der Ausdruck der überreizten Sexualität.

Im letzten Drittel des 19. Jahrhunderts setzte auf der Basis der damals herrschenden Streitfragen, die manche Übereinstimmung zeigten, unterstützt durch die verbesserte histologische Technik eine vermehrte objektive Beschäftigung mit der Klärung der intrauterinen Veränderungen ein, in deren Folge es zur Regelblutung kommt (Leopold 1878). Weil die Frage der Beschaffenheit des uterinen Oberflächen- und Drüsenepithels um die Zeit der letzten Jahrhundertwende noch nicht gelöst war und zu Widersprüchen führte, versuchte Böhme (1909) dieses Problem bei den Haussäugetieren einer Lösung näherzubringen. So schilderte auch Minot (1889) den durch Hyperaemie und Hyperplasie des endometralen Bindegewebes sowie Hypertrophie der Uterindrüsen gekennzeichneten menschlichen Uterus, wobei es zur Lockerung des oberen Viertels der Mucosa uteri kommt, die ohne Aus- 
bildung von Deciduazellen abbricht. Erst durch die Befunde von F. Hitschmann und L. Adler (F. H.: 1870-1926; L. A.: 1876-1958) (1908) zum Bau der Uterusschleimhaut des geschlechtsreifen Weibes wurde zu Beginn des 20. Jahrhunderts der mensuelle Zyklus in ein neues Licht gerückt, obwohl beide Autoren in ihm eine «chronische Endometritis» sahen. Diese irrige Diagnose wurde später durch die intensiven Untersuchungen von R.Schröder (1884-1959) (1914, 1930) korrigiert. Deutlich bewies C.G. Hartman (1932) die Identität des weiblichen Sexualzyklus bei der Frau mit dem der catarrhinen Affen, nachdem schon Wirsung (1572) entgegen der Überlieferung angegeben hatte, auch die Äffin menstruiere. Im Altertum und bis in die Neuzeit wurde angenommen, die Frau sei das «solum animale menstruale» (Müller-Hess). Sehr sorgfältig und umfassend konnte G. W. Bartelmez (1933) den menschlichen Monatszyklus auf histo-morphologischer Basis erläutern. Im Menstrualblut sehen wir das Ende der zyklische ablaufenden, morphologischen Veränderungen der Gebärmutter, die der Vorbereitung des Biotops für eine Keimeinnistung und der Placentation dienen (Strauss 1986).

Ohne weiter auf die historisch differenten Betrachtungen über Aufgabe und Herkunft des Periodenblutes einzugehen, seien aus diesem Sektor der historischen Placentologie noch einzelne wesentliche Beobachtungen erwähnt, die uns auch heute noch beschäftigen. So deutete bereits Aristoteles an, daß es auch bei einer Gravida zu einer Regelblutung aus der Vagina kommen könne. Ein solches Ausnahmeereignis, das keinesfalls ein Abort sein muß, sehen wir, entsprechend der Situation, nicht unbedingt als ein signum mali ominis an; wir wissen heute, daß der Genitalzyklus während der Schwangerschaft verdeckt weiterläuft (Strauss 1986). Sobald beim Homo sapiens der Höhepunkt der Luteinphase überschritten ist, setzen etwa um den 24.Zyklustag mit und ohne Schwangerschaft in der Gebärmutter gewisse, noch heute als Rückbildungsvorgänge gedeutete Reaktionen ein. Dabei erfolgt trotz Keimeinbettung um die Zeit der ersten ausbleibenden Monatsblutung nicht selten ein kurzer, als Implantationsblutung bezeichneter Blutaustritt. Nach Minot sind die uterinen Veränderungen während Menstruation und Schwangerschaft homolog; der mensuelle Zyklus werde durch die Gravidität nur verlängert und umgebildet. Diese Veränderung findet während des Zyklus statt, denn das Ovum kann die mensuellen Veränderungen nur modifizieren, jedoch nicht zur Bildung der Decidua anregen. In diesem Zusammenhang wird es auch verständlich, daß bereits im letzten Decennium des 16. Jahrhunderts mensuelle Beschwerden mit den Schwangerschafts-Molimina identifiziert wurden. 


\section{Ovulation und Menstruation}

Schon 1671 gab Theodor Kerckring, der Vater der Kerckringschen Falten (= Plicae circulares) im Dünndarm an, daß bei Ehe- und Jungfrauen sowohl in der praemenstruellen Phase als auch bei sexueller Erregung, was in diesem Jahrhundert im Sinne der provozierten Ovulation im Gegensatz zur spontanen Ovulation von H. Stieve (1886-1952) (1943) nachdrücklich postuliert wurde, eine ohne Gravidität meist nicht erkannte Ovulation erfolge. Damit wird klar, daß schonvor mehr als 300 Jahren ein gewisser biologischer Zusammenhang zwischen Eisprung und mensuellem Zyklus angenommen wurde. Bereits 1672 war Ch. Germann der Auffassung, daß der Ovulationsrhythmus mit dem Menstruationszyklus conform sei (Müller-Hess). Diese Beziehung war zu Beginn des 19. Jahrhunderts als Kulturerscheinung mißdeutet worden. Noch um Mitte des letzten Jahrhunderts war man der Ansicht, die Monatsblutung gehöre nicht unbedingt zum weiblichen Genitalzyklus; sie sei nur eine Abart der letzten Phase, quasi seine Crisis, die man seinerzeit vergleichend und gar nicht unzutreffend mit Mauserung bezeichnete.

\section{Eihäute}

Obwohl es seit Galen bekannt war, daß das sog. $E i$ aus mehreren einander einschließenden Häuten besteht, war man sich lange über deren Zahl nicht einig: einmal wurden zwei, ein andermal drei Häute (1.Decidua und Chorion, 2.Amnion und 3. Dottersack genannt, denen als 4. Hülle noch die Allantois zugefügt wurde. Erst durch den Berner Albrecht von Haller (17081777) und W. Hunter (1718-1783) (Needham 1959) erfuhr man, daß Zahl und Beschaffenheit dieser sog. Eihäute oder Fruchthüllen je nach Schwangerschaftsgrad verschieden sind.

Die erste dieser Hüllen ist die Membrana caduca des Uterus oder Huntersche Haut (Membrana decidualis = hinfällige Haut). Bei ihr handelt es sich um eine sowohl zyklisch als auch durch die Gravidität bedingte morphologische Umbildung der Gebärmutterschleimhaut mit entsprechend alternierenden Aufgaben. Eine genaue Beschreibung der Decidua verdanken wir J. F. Lobstein (1804), der um die Wende des 18./19. Jahrhunderts Prosector an der Medicin-Schule in Straßburg war; er war auch der Auffassung, das Chorion (= Zottenhaut), ein Produkt der Frucht, bilde einen Teil der Membrana caduca, die gemeinsam Ei und Mutter angehöre. 
Als erster stellte John Hunter (1728-1793) (1835/37) eine zutreffende Hypothese über die Herkunft der Decidua auf, wobei Aristoteles ebenso wie Aretaios von Cappadocien schon lange vor ihm dieses Fachwort gebraucht haben sollen (De Witt 1969; Velpeau 1833). Hunter erklärte, die Decidua sei ein endometrales Produkt, was nicht überall akzeptiert wurde. Sein Bruder William (1718-1783) nahm an, die Membrana decidualis sei die entzündete Uterusschleimhaut (s. Hitschmann und Adler), die keine Umwandlung erleide. Trotzdem wandte er sein Augenmerk den decidualen Reaktionen und ihren Veränderungen in graviditate zu, so daß er daraus die Begriffe Membrana decidualis vera und reflexa ableiten konnte. So kommt ihm das Verdienst zu, diese Differenzen der hinfälligen Haut getroffen zu haben, die bald von den meisten Anatomen und Physiologen anerkannt wurden. Ercolani (1868) dagegen sah in der Decidua eine Neubildung und keinen direkten Abkömmling der Mucosa uteri. Direkte Erben dieser Ansicht waren L.Oken (1779-1851), J.C.G. Joerg (1779-1841) (1821), der als erster die Basis für die Physiologie der Geburt legte, und C.E. von Baer (1792-1876) (1828), der erst nach Stenos und de Graafs Vorarbeiten das Säugerei entdeckte (1827). Ergänzend zu Hunter beschrieb Bojanus (1776-1827) einen dritten Deciduaabschnitt, die Decidua serotina oder basalis. So herrschte schon zu Beginn des 19. Jahrhunderts im Prinzip Klarheit über die morphologische und topographische Unterteilung dieser mütterlichen Membran, obgleich Joerg und Seiler (De Witt) eine Decidua capsularis ebenso wie von Baer ablehnten. Oken und Velpeau erkannten sie vom Vergleichsstandpunkt aus nur für den Menschen. Wahrend K. F. Burdach (1776-1847) (1835) und Velpeau ohne Vergleiche zur oviparen Tierwelt annahmen, die Decidua diene allein der Keimeinnistung, vertrat Breschet (1833) die noch heute gültige Ansicht, die Decidua funktioniere nicht nur als Einpflanzungsort, sondern bewahre die Mucosa uteri auch vor Flüssigkeitsverlust und diene so dem foeto-maternen Austausch. Velpeau bezeichnete die Siebhaut mit Membrane anhiste; er hielt sie für nicht vaskularisiert und sprach ihr deshalb jegliche Ernährungsaufgabe ab. Die physiologische Bedeutung der Decidua war in jenen Jahren noch immer umstritten, da auch ein so bedeutender Naturforscher wie G.Cuvier (1769-1832) (1817) der Annahme huldigte, sie entspreche der Hühnereischale.

Mit Chorion bezeichnen wir die sich aus Chorionmesoderm und Trophoblast (= Nährblatt) aufbauende, mittlere Eihaut; sie differenziert sich aus dem zunächst einschichtigen Trophoblasten, der als äußerste Fruchthülle und Abkömmling des Keimes mit dem Endometrium in Kontakt steht. 
Obwohl von Baer (1828) glaubte, im Discus oopherus (= ovigerus) des Eies das zukünftige Chorion, also eine Eihülle, vor sich zu haben, gelang es ihm, die Entwicklung der Fruchthüllen mit richtigem Verständnis zusammenzufassen. Der bekannte Münchner Anatom und Physiologe Th. L. W. Bischoff (1807-1882) (1842) konnte jedoch die Discus ovigerus-Zellen vom Ovum entfernen, das er als Keimblase ( = Vesicula blastodermica) bezeichnete, und somit zeigen, daß jene Zellen nicht das zukünftige Chorion repräsentieren. Auf dieser Vorstellung basiert auch Bischoff's Theorie, die Außenschicht der Zottenhaut sei, weil sie in direktem Kontakt mit dem mütterlichen Blut steht, ein Abkömmling der Mucosa uteri. Entschiedenen Auftrieb erfuhr die Klärung des Chorionaufbaues durch die Forschungen von Langhans.

Nach Ansicht von Lobstein war die uterine Innenfläche, die Membrana caduca, von einer schleimigen, fest mit dem Uterus vereinigten Haut überzogen, die Fortsetzung der Scheide sei. Er war weiter der Ansicht, das Chorion dringe schon, bevor die Placenta vorhanden sei, mit verzweigten «Fäden» in die Huntersche Haut ein. Sabatier (1777) glaubte, die Membrana caduca als Produkt der Schwangerschaft gehe bei der Geburt, wie durchaus richtig beobachtet, mit der Frucht ab. Aufgefallen war damals bereits der Reichtum an spiralisiert verlaufenden mütterlichen Gefässen in der Membrana caduca. Heute ist es durchaus verständlich, daß vor bald 200 Jahren Caduca und Chorion mit fortschreitender Gravidität tiefer und vermehrt in die Decidua eindringen und so zum sukzessiven Aufbau des Mutterkuchens führen. Heftig umstritten war seinerzeit auch der Blutgefäßgehalt des Chorions; wie J. F. Blumenbach [(1752-1840), Prof. der Anatomie in Göttingen] (1781), sah auch Haller keine Gefäße, während H.A. Wrisberg (1739-1808) (auch in Göttingen) (Kölliker 1884) sie als die «Fäden» erkannte.

Etwa in der Mitte des 19. Jahrhunderts vermochte E. von Beneden (1846-1910) (1875) im Keim embryonales Ectoderm und Trophoblast, die choriale Epithelschicht, zu separieren (Grosser 1909 a). Mit dieser Trennung war jedoch das Problem des zweischichtigen Trophoblasten, an dem wir heute Plasmoditrophoblast ( = Syntrophoblast Arey 1959) und Cytrotrophoblast (Langhans 1882) unterscheiden, noch nicht gelöst, denn auch Kölliker huldigte mit anderen Untersuchern der Überzeugung, die Außenschicht der Chorionzotten sei mütterlicher und die innere Epithellage (= Endochorion), die später verschwinde, embryonaler Herkunft. Dieser Auffassung stimmte anfangs auch Langhans trotz der durch ihn erfolgten wesentlichen Klärung der Trophoblastverhältnisse zu. 
Nach Ercolani sollte das Epithel der Chorionzotten am Anfang und am Ende der Schwangerschaft von verschiedener Bedeutung sein. Anfänglich gehöre es dem Embryo bzw. Foetus und später der serösen Hülle an, so daß es nur einen Teil der Placenta bilde. Zwischen diesen beiden Zuständen sollte ein Mittelzustand vorhanden sein. Dieser der damaligen Zeit gut entsprechenden Beobachtung konnte Kölliker nicht zustimmen.

Auf das Chorion folgt nach innen das Amnion (Schafshaut). Zwischen Chorion und Amnion sah Kölliker eine gallertige Masse, die er Membrana intermedia und Velpeau Magma réticulé nannte. Diese Zwischenschicht wurde je nach Situation entweder als eine eingedickte eiweißhaltige Flüssigkeit oder als gallertige Bindesubstanz angesehen. Zu Beginn des 19. Jahrhunderts war der Gefäßgehalt des Amnions umstritten. Man war damals der Auffassung, das menschliche Amnion besitze im Gegensatz zu dem der Vierfüßer keine Gefässe. So war auch nicht klar, woher das Fruchtwasser, der Liquor amnii, kommt. Im Gegensatz zu den damaligen Physiologen, die im Fruchtwasser ein foetales Produkt (Schweiß, Urin usw.) sahen, nahm Haller an, es stamme vom Uterus (Lobstein). Tatsächlich wird es von der Amnionwand sezerniert.

Valentin (1835) schreibt Riolan dem Jüngeren (1577-1657), dem Professor der Anatomie und Botanik in Paris, die Beobachtung zu, als erster den Dottersack (= Saccus vitellinus), der den Embryo nicht umhüllt, bei den Säugern erkannt zu haben. In den letzten Jahren des 3. Decenniums des 19. Jahrhunderts wurde dann der Zusammenhang des Darmes mit dem Dottersack durch von Baer und J. Müller (De Witt) erkannt. Infolge dieser Verbindung dient der Dottersack in der ersten Zeit der Gravidität der Aufnahme uteriner Sekrete.

Mit der Diskussion über die Herkunft des Chorions als Eihaut war auch die Bedeutung der Allantois, der «wurstähnlichen Haut», verknüpft. Das Wissen um die Allantois reicht weit zurück ins Altertum. Unklar war allerdings lange ihre Aufgabe. An der menschlichen Placenta hat N. Hoboken, von dem 1669 eine wertvolle Arbeit über die Placenta erschien, nochmals die Allantois entdeckt und nannte die zwischen Chorion und Amnion gelegene Blase Membrana urinaria; trotz der Namengebung konnte er ihre Funktion nicht deuten. Bei dieser Unkenntnis wurde sie anschließend als Membrana Hobokenii beschrieben. Erst J.F. Meckel der Jüngere (1781-1833) (Enkel von J.F. Meckel dem Älteren) (Prof. der Anatomie in Halle) (1820) bestimmte diese «mittlere Membran» als Allantois, deren Funktion etwa gleichzeitig durch Untersuchungen am «Harnsack» des 
Hühnerembryos geklärt werden konnte. Haller lehnte als zu seiner Zeit moderner Physiologe und unter meist falschen Schlüssen die placentare Funktion zunächst im Gegensatz zu verschiedenen Anatomen das Vorhandensein einer Allantois $a b$, die auch vom Holländer B.S.Albinus (1697-1771), durch seinen anatomischen Atlas bekannt, im 18. Jahrhundert (1748) beschrieben wurde (Lobstein). Später gestand Haller seinen Irrtum, nachdem er sich anhand der Untersuchungen zahlreicher Autoren von ihrem Vorhandensein überzeugen ließ. Blumenbach und Th. Soemmerring (1755-1830) (ebenfalls in Göttingen) (1799) hatten die Allantois als beständigen, zur Organisation der Frucht gehörigen Teil erkannt. Nach ihrer übereinstimmenden Auffassung hatten alle warmblütigen Tiere eine identische Blase, die man seinerzeit bei Homo Vesicula umbilicalis, bei den Vögeln und den Säugetieren Allantois nannte. Blumenbach und Soemmerring waren ferner der Meinung, der Allantoisinhalt trage zum Wachstum des Foetus bei, da sie den Blaseninhalt mit dem Eidotter verglichen. Trotz dieser Feststellungen in Göttingen war Bischoff von der Allantois als einem selbständigen Organ nicht überzeugt. Ebenso vertrat W. His (1831-1904), einer der Gründer der Entwicklungsmechanik, die Ansicht, der menschliche Embryo habe keine Allantois; er nannte dieses Gebilde Bauchstiel, der mit dem Chorion verbunden sei (Kölliker). Obwohl nicht entschieden, ob der Bauchstiel = Allantoisstiel, der Urachus, die Blasenschnur, ein echter Kanal ist, fand ihn schon Haller in möglicher Verbindung mit der Harnblase. Generell war man damals der Ansicht, daß die Allantois mit Ausnahme beim Menschen bei allen Säugern Urin aufnehme. Lobstein vertrat immerhin die Auffassung, Allantois und Saccus vitellinus seien nicht nur ihrer Natur, sondern auch entsprechend ihrem Nutzen einander gleich. Ebenso war Haller zunächst der Meinung, der Dottersack sei ein Teil der Allantois. Diese beiden Organe wurden früher häufig verwechselt, weshalb das Fachwort Vesicula umbilicalis sowohl für das eine als auch für das andere Organ gebraucht wurde. Durch seine Verbindung mit dem Urogenitalsystem, d.h. mit den Ausführungsgängen der Wolffschen Körper, den Urnieren, glaubte man, dieser sog. embryonale Harnsack erhalte die stickstoffhaltigen Zersetzungsprodukte des embryonalen Stoffwechsels. Die Allantois dient als Atmungs- und Resorptionsorgan.

Den verschiedenen Interpretationen der Eihäute ist G.G.Valentin (1810-1883) (1835), der einstige Berner vergleichende Anatom und Physiologe, ausführlich nachgegangen, wobei er auch die zahlreichen, heute zum Teil nicht mehr gebräuchlichen Fachausdrücke aufführt. Valentin schließt 
seine detaillierten Ausführungen: «Die Hauptfrage bleibt vielmehr die, wer sie (die Eihäute, F.St.) zuerst völlig und richtig gesehen, in all ihren Verhältnissen beobachtet und erkannt habe. Zweitens muß man bedenken, daß alle älteren Beschreibungen von Eihäuten in gewissem Grad für uns unverständlich, wenigstens nicht sicher zu deuten sind, da zu jener Zeit mancher gerade die Genauigkeit in der Anatomie darin zu finden glaubte, ein Organ oder einen Organtheil in recht viele Membranen, und seyen diese noch zu sehr erkünstelt, zu zerfällen. Auch wechseln die Ausdrücke auf die freieste Weise, so daß schon zu dieser Zeit der älteren Werke der Undeutlichkeiten genug existieren.»

\section{Implantation}

Verständlicherweise ist bei Betrachtungen über die Placenta auch das Problem der Implantation zu erwähnen. Zu Beginn des 19. Jahrhunderts äußerte 1820 J. F. Meckel der Jüngere die Ansicht, die Decidua bedecke das Ostium internum cervicis. Sowohl E. Sandifort (1742-1814) (1777) als auch Blumenbach widersprachen dieser Auffassung und verneinten eine deciduale Überdeckung sowohl der Tubenmündungen als auch des Gebärmutterhalses. Im Rahmen der Vorstellungen von Meckel vertrat Burdach die Theorie, der Keim stülpe sich von hinten, d.h. von der Uteruswand her, in die Decidua ein, die nach Auffassung der meisten Beobachter bei der Geburt mitausgestossen werde. Der Ansicht der retrograden Keimeinnistung trat Bischoff (1834) energisch entgegen. In jenen Jahren standen sich zwei grundsätzliche Theorien über die Keimeinnistung gegenüber. Die eine war die Burdachsche Auffassung der Einstülpung (= Inversion), bei der sich der Keim, ohne das Cavum uteri zu erreichen, zwischen den Decidua-Schichten festsetzen würde. Diese Vorstellung wurde von Bojanus, Nägele und Velpeau (Bischoff) unterstützt.

Die andere Ansicht, welche Valentin nachdrücklich vertrat, war die Theorie der Einsaat (= Propulsion), die abgesehen vom Abdrängen der Decidua duch den Keim, weitgehend der derzeitigen Auffassung der durch Histolyse bedingten Einpflanzung entspricht. Valentins Ansicht von der Keimeinnistung basiert, wie er selbst angibt, auf den Vorstellungen von Carus, Heusinger, W. Hunter und Meckel dem Jüngeren. Der von Valentin vertretenen Theorie stimmten mehrere Forscher zu, so vor allem der Zürcher Bürger R.A.Kölliker (1817-1905), der von 1847 bis zu seinem Tod in 
Würzburg unter spezieller Berücksichtigung der Gewebelehre als Anatom tätig war. Auch Minot bejahte Valentin; beide Embryologen waren sich darüber einig, daß schon vor der Implantation ein decidualer Zelltypus existiere. Den Beweis der praegraviden und praemenstruellen Decidualisierung lieferten Denman und Evrat (Hyrtl 1884). Minot vertrat die Auffassung, die Entwicklung einer praemenstruellen und praegraviden Decidua sei eine durch die Fernwirkung eines Eies ausgelöste Umbildung des Endometriums. Zu Ende des 19.Jahrhunderts äußerte sich dann auch Peters (Geburtshelfer in Wien) (1899) über die Einbettung des menschlichen Eies.

\section{Placenta}

Der Begriff «Placenta», der bis zur Mitte des 16. Jahrhunderts in der Anatomie nicht vorkommt, ist eine Wortfrucht des griechischen Sprachstammes; er wurde von dem Anatomen Colombo (1516-1559) geschaffen; er sah in der Nachgeburt einen parenchymatösen Körper von Kuchengestalt (Hyrtl 1884). Hier ist festzuhalten, daß zu Beginn des letzten Jahrhunderts der Inhalt der schwangeren Gebärmutter unter Ausschluß der Placenta Ei genannt wurde; man sprach von den Häuten. Unter Ei verstand man nur den häutigen Sack mit Embryo bzw. Foetus und die zugehörige Flüssigkeit. Aristoteles bezeichnete das erste Zeugungsprodukt aller Organismen als Keim. Falls in diesem Körper neben der Anlagesubstanz auch das erste Nährmaterial für den Embryo miteingeschlossen ist, war das für ihn ein Ei (Bloch 1904), ein Begriff, den auch Kölliker noch gebrauchte.

Im 18. Jahrhundert leisteten mehrere Forscher mit ihren Studien wesentliche Beiträge zur Kenntnis der weiblichen Geschlechtsorgane. Die bedeutendsten Autoren waren William Hunter $(1774,1794)$ und sein Bruder John; in ihren Untersuchungen standen Uterus und Placenta im Vordergrund, wobei sie sich speziell mit der placentaren Blutzirkulation befaßten. J. Hunter verfolgte injizierte Arterien und Venen aus der Uteruswand in den Fruchtkuchen, womit er das eingespritzte Material durch die Gefäße in den intervillösen Raum brachte, den er «the spongy cells of the placenta» nannte. Diese Beobachtung übernahm sein Bruder in die Schilderung des schwangeren Uterus, worin er besonders auf die endometralen Spiralarterien unter Beigabe einer Abbildung und ihre Beziehungen zum kindlichen Kreislauf hinwies; der erwähnten Veröffentlichung fügte er die ausgezeichnete Abbildung eines Uterus gravidus (mens V) bei (Abb.3) (Strahl 1906). 
Damit hinterließ W.Hunter eine klare Beschreibung des menschlichen Mutterkuchens in utero. Im «Uterus gravidus» erkannte und interpretierte William auch den Dottersack (Ramsey 1982).

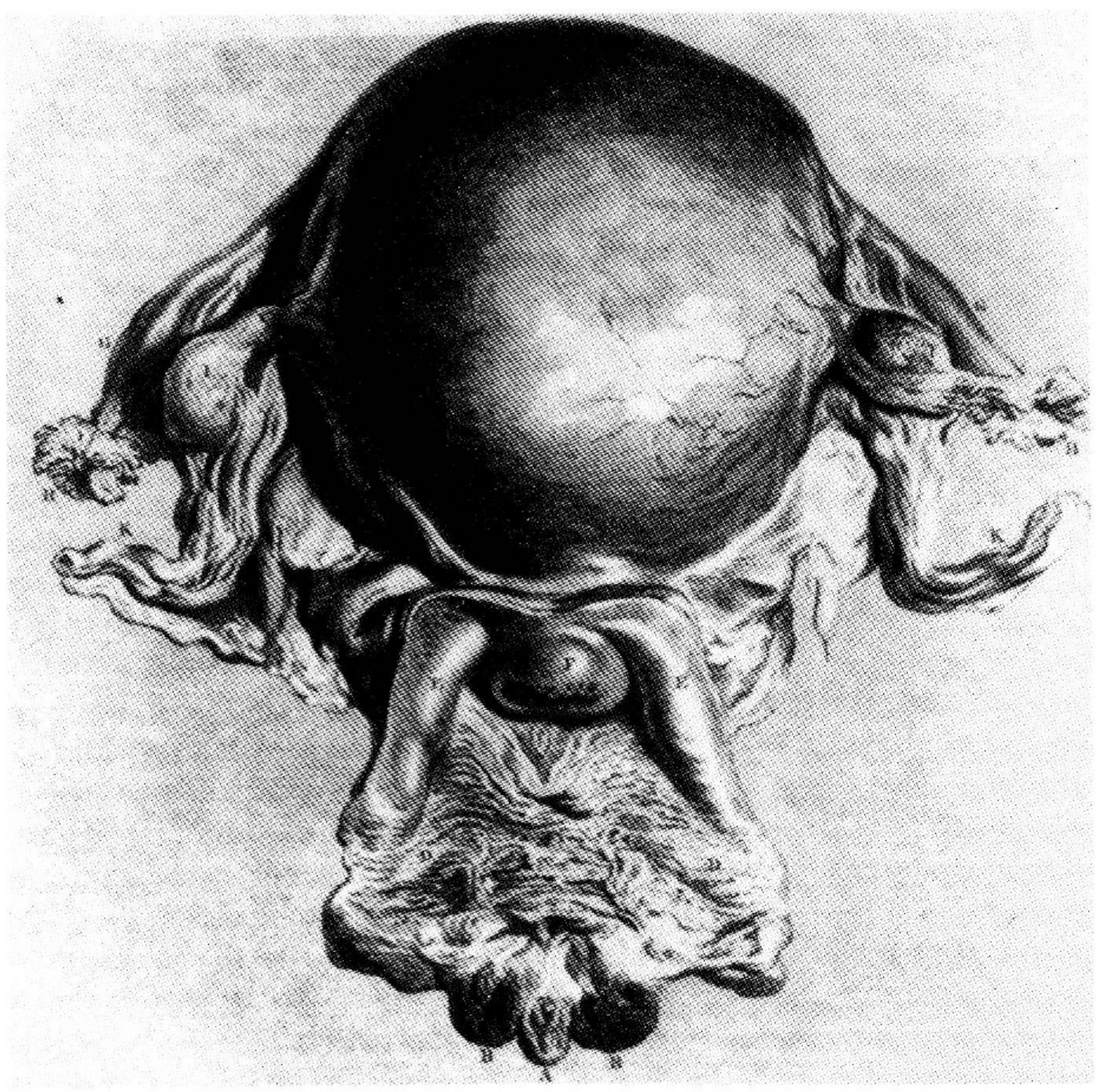

Abb. 3 Uterus gravidus mens V nach W. Hunter (aus Ramsey 1973)

Mit dem alten Begriff Cotyledo oder Cotyle, der nicht ganz mit unserem heutigen Fachwort identisch ist, verband man einst die Vorstellung uteriner Brustwarzen, die mit der von Galen vermuteten Gefäßverbindung Mammae-Uterus verknüpft seien (Bloch). Vor gut 100 Jahren bezeichneten 
Anatomen und Geburtshelfer mit Cotyledonen die zu einem Kuchen vereinigten Lappen der Placenta, die wir heute Placentone (Schuhmann 1981; Schuhmann, Borst, Geier, Kraus 1977) nennen. Es ist widersprüchlich Cotyledo für Lappen zu gebrauchen, denn Cotyledo bedeutet Pfanne bzw. napfförmig (Hyrtl 1884). Diese Interpretation geht auf die in der Gebärmutterschleimhaut der Wiederkäuer vorhandenen napfförmigen Erhebungen, die Carunculae, zurück. Unter Cotyledonen verstehen wir die zu Bündeln zusammengefaßten gefäßhaltigen Chorionzotten. Mit Cotyledonen interpretierte man zu Beginn des 19. Jahrhunderts noch die einzelnen Placentarlappen (Placentone) und -läppchen, während wir heute damit die feineren Aufzweigungen des foetalen Placentaranteiles bezeichnen. So sah man den Mutterkuchen aus mehreren Portionen aufgebaut, die sich beim Menschen zu einem Organ vereinigen, jedoch beim Tier je nach Gattung einzeln an der inneren Uterusoberfläche befestigt sein können. Unbewußt war damit schon die Vielgestaltigkeit der Nachgeburt aufgefallen und der Grundstock einer vergleichenden Placentologie geschaffen. Mit der detaillierten Untersuchung erkannte man auch die Aufzweigung der Nabelgefässe auf der kindlichen Placentaroberfläche und ihr Eindringen in die Cotyledonen, wo sich jene feiner aufzweigen. Die ursprünglich als «Fäden» des Chorions beschriebenen Fortsätze erkannte man jetzt als Blutgefässe.

Mit der Erörterung des Baues des Chorions und damit des Trophoblasten war auch die Frage nach der Herkunft der chorialen Blutgefässe verknüpft. Von Baer und Valentin vermuteten sie als Derivate des Endometriums. Die Lösung dieses Problems ergaben erst die Untersuchungen von A.T. Hertig (1935); er erkannte die Vasogenese im Chorionbindegewebe.

\section{Blutversorgung von Placenta und Kind}

Mit der Deutung der Fäden als Blutgefäße begann die Diskussion über den Blutumlauf zwischen Mutter und Placenta. Sehr bedeutend ist hier die funktionelle Auffassung von G. C. Arantio (1530-1598); er verglich die seiner Ansicht nach schwammige Placenta, ihres Gewichtes und ihres Gefäßreichtums wegen mit der Leber. Er nannte den Mutterkuchen sofort Hepar uterinum und schrieb ihm die dynamische Funktion des Haematopoeticums und der Blutreinigung zu (Bloch). Gegen Ende des 17. Jahrhunderts sah C. Bontekoe (1685) eine Vorbereitung auf eine Gravidität im zyklischen Auswachsen mit Zweigbildung der endometralen Arterien. Die Zweige 
sollten sich später mit den Blutgefäßen der kindlichen Placenta vereinigen. Kommt es jedoch nicht zu einer Schwangerschaft, so öffnen sich diese Äste und bluten so lange, bis sie sich von selbst einkrampfen und die Blutung aufhört (Periodenende) - wie das schon Galen erklärt hat (Müller-Hess).

J.Freind (1675-1728), der erste englische Medicinhistoriker, vertrat 1728 die Auffassung, das die Placenta durchströmende Blut sei Menstruationsblut. Einzelne Autoren, wie z.B. Haller, nahmen eine Verbindung zwischen mütterlichen und placentaren Blutgefäßen an, während andere Forscher wie Hunter und Lobstein eine unmittelbare Verbindung bestritten und Saftübertragung durch Absorption vermuteten; dafür fehlte allerdings damals der erforderliche Beweis. Lobstein deutete doch immerhin den materno-placentaren Kreislauf wie folgt an:

«Solange das Kind in der Gebärmutter enthalten ist, geschieht in dem Mutterkuchen eine doppelte Circulation; die eine hängt vom Foetus ab; die andere von der Mutter, und durch sie wird ihr Blut in die Zellen und zwischen den Nabelgefässen befindliche Räume ergossen.»

F. D. Herissant (1714-1773) (1743) wies darauf hin, daß das Blut der Umbilicalvenen arteriell sei; deshalb nahm er in Analogie zu den Lungenvenen an, die Placenta habe nur respiratorische und keine ernährenden Aufgaben und nannte sie Pulmo uterinum.

Der Berliner Anatom, Botaniker und Geburtshelfer J.F. Meckel der Aeltere (1714-1774) (erster Lehrer an der neuen Hebammenschule der Charité) war der Ansicht, dem Kind werde durch die Gefäße der Mutter Blut eingespritzt. Erst allmählich reifte so die Erkenntnis, daß foetaler und mütterlicher Kreislauf getrennt sind.

\section{Ernährung von Embryo und Foetus}

Über dieses Problem wurde für gut 2000 Jahre diskutiert. Noch Harvey war der Ansicht, die Ernährung erfolge durch das Fruchtwasser. Etwa gleichzeitig bestritt 1711 C. Falconnet (1671-1762) die Annahme, das mütterliche Blut sei die embryo-foetale Nahrungsquelle (Bloch; Needham). Auch Haller huldigte aufgrund seiner konservativen Einstellung der Auffassung, der Liquor amnii werde vom Kind per os aufgenommen. Die hippokratische Theorie, die Amnionflüssigkeit diene der Atmung und Ernährung des Embryos, hatte demnach in verschiedenen Variationen lange Gültigkeit. Im 
Rahmen einer Ernährungsaufgabe hatte schon Aristoteles dem Menstruationsblut den eigentlichen und einzigen Beitrag der Frau für den Aufbau eines Kindes zugesprochen (Bloch; Hyrtl 1884). Hippokrates vermutete in den Menses eine Beziehung zur Muttermilch. Auf dieser Annahme postulierte dann Galen eine Gefäßverbindung zwischen Brustdrüsen und Uterus. Harvey vertrat aufgrund seiner Placenta- und Decidua-Untersuchungen an Ungulaten auch die Auffassung, die Caduca sei eine eitrige Masse, wie das nach ihm ähnlich auch noch Hitschmann und Adler annahmen. Das Secret des Mutterkuchens interpretierte er als «uterine Milch», die via Fruchtwasser und Nabelstrang dem Foetus zugeführt werde. Bedeutend war auch Harveys Atmungstheorie: da die Frucht bis zur Geburt ohne Luft lebe, müsse sie, falls sie bei der Geburt nicht sofort atme, durch Kompression der Nabelschnur sterben. Im ersten Viertel des 18. Jahrhunderts wurde unter dem Einfluß des zu Zeiten Hallers aufgekommenen Vitalismus lebhaft über Aufgabe und Bedeutung der Regelblutung diskutiert; dabei wurde der Gebärmutter eine Blut-Plethora zugesprochen, die für die intrauterine Kindernahrung wesentlich sei. Diese Auffassung wurde von der Vorstellung beeinflußt, der Foetus werde mit Chylus (Chylus-Plethora) versorgt (MüllerHess). Die verschiedenen Theorien der embryonalen Ernährung in den einzelnen Zeitabschnitten bespricht Needham sehr ausführlich.

Auch Lobstein versuchte, die Ernährung des Foetus zu klären. Dazu führte er einleitend aus, daß die zu seiner Zeit entsprechenden Ansichten vertreten würden, ohne die der foetalen Ernährung dienenden Grundlagen zu kennen. So wurde noch im ausgehenden 18. Jahrhundert verschiedentlich angenommen, daß 1. das Fruchtwasser der Ernährung diene, obwohl man nicht wußte, woher es kommt, 2. ohne die Verbindung MutterkuchenGebärmutter genau zu kennen, vermutete man in dieser Gemeinschaft eine Ernährungsquelle, und 3. in den nur angenommenen Lymphgefäßen des Nabelstranges Lieferanten. Auf dieser Basis setzte sich Lobstein mit den Ernährungsmöglichkeiten für den Foetus auseinander. So erläuterte er eingehend die Gründe, die ihn bewogen, das Fruchtwasser als Nährstoff anzunehmen. Nicht weniger wichtig war ihm die Placenta, deren entsprechende Aufgabe sich mit steigender Schwangerschaftsdauer in positivem Sinn wandelt. Auch das Nabelbläschen (Dottersack) diene seiner Meinung nach aufgrund einer gewissen Ähnlichkeit zu den Cotyledonen der Pflanzen der foetalen Ernährung.

Zu Beginn des 19. Jahrhunderts zeichnete sich langsam eine Abkehr von der Fruchtwasser-Theorie ab. 1837 vermutete Eschricht, wahrscheinlich auf 
der Basis der Vorstellung, die Placenta habe nur eine respiratorische Aufgabe, die Glandulae utriculares seien die Nährstofflieferanten (Bischoff 1842). Erst Hyrtl (1884) und Kölliker haben energisch der Ansicht, der Liquor amnii sei die foetale Nahrungsquelle, widersprochen, da der geringe Proteingehalt und die alkalische Reaktion für die Ernährung ungeeignet seien. Die Herkunft des Fruchtwassers schrieb Kölliker aufgrund seiner Beobachtung an Reptilien- und Vogeleiern unwiderleglich dem Foetus zu; als Quellen vermutete er sowohl die Haut als auch die Nieren. Trotz dieser Ansicht wagte er nicht in Abrede zu stellen, daß «nicht auch der mütterliche Organismus an der Bildung des Fruchtwassers sich beteilige», wobei "vor allem an die reichlichen Gefässe der Decidua vera zu denken sei». Entschieden fortschrittlicher und den tatsächlichen Verhältnissen praktisch entsprechend ist die Aussage von J. Müller, die foetalen Blutgefäße ziehen die «Säfte der Mutter an» und diese können so «direkt ins Blut des Foetus» eindringen (Spanner 1936). Wesentliche Beobachtungen liessen schon Bischoff (1834) erkennen, daß die Sauerstoffzufuhr zum Embryo und Foetus durch die Nabelschnurgefäße erfolge.

\section{Placentologen}

Entschiedenen Auftrieb erfuhr die Klärung des Chorionaufbaues durch die Forschungen des ehemaligen Berner Ordinarius für Pathologische Anatomie (1872-1915) Th. Langhans (1839-1915), der nicht nur Placentologe, sondern auch ein bedeutender Nephrologe war. 1882 publizierte er die bekannte Arbeit «Über die Zellschicht des menschlichen Chorions», mit der er seinen Namen für alle Zeiten mit der von ihm entdeckten Zellschicht des Chorionepithels, den Langhans-Zellen, dem heutigen Cytotrophoblasten (Langhans layer, Langhans cells), verband. Er vermutete, daß diese Schicht sich aus der Hautplatte der serösen Hülle entwickle.

Auch hier griff Kölliker ein und schrieb der «Langhansschen Zellschicht» eine verschiedene Bedeutung zu; er sah stellenweise in ihr einen Teil der Bindesubstanz, weil sie in der Frühphase einen deutlichen Zellcharakter hat, jedoch im zweiten Trimenon mit Ausnahme einzelner verdickter Stellen, wie lange vermutet, nicht mehr zu erkennen sei. Bei entsprechender Fixation und Färbung läßt sich jedoch der Cytotrophoblast während der ganzen Gravidität nachweisen. Es kommt nur zu einem starken Schwund im zweiten Trimenon (Strauss 1967, 1982). 
In seiner ersten placentologischen Arbeit (1870) besprach Langhans die Histologie der Decidua und diskutierte die Herkunft der Deciduazellen; damals folgerte er, daß eine Entscheidung über die Frage der Zugehörigkeit dieser Elemente noch nicht gegeben sei. Über die «Gegenseitige Verbindung der Placenta foetalis und materna» griff er in der gleichen Publikation das Problem der Haftzotten und der sog. choriodecidualen Gefäße auf. Dabei war er der Meinung, die Verbindung der kindlichen und mütterlichen Placentarteile werde «in der Art hergestellt, daß die Zotten der Placenta foetalis ... in das Gewebe der mütterlichen Placenta sich einsenken, daß ein kräftiger Zug an jener nie diese Verbindung löst, sondern immer eine Zerreißung im mütterlichen Gewebe verursache (1877). Eine eingehende Beschreibung des histologischen Befundes finden wir auch für die pilzförmig sich verbreiternden Haftzotten, wobei das Zottenende epithelfrei ist, und die kindlichen Gefäße «reichen in dem freien Zottenstamme nur bis an die Placenta materna ... oder in den von der Placenta materna umschlossenen Theil verschieden weit hinein» (ein sehr wichtiger Befund!). Eine durchaus ähnliche Schilderung gab schon Ercolani von den Haftzotten, die Kölliker Haftwurzeln nannte und sie epithelfrei bis in die Placenta uterina verfolgen konnte. Der von Langhans vermuteten Verbindung von Zotten und Decidua folgen wir heute jedoch nicht mehr. Weiter unterschied er an den Epithelfortsätzen der Zotten sowohl frei endende als auch Übergänge von einer Zotte zur anderen. 1872 besprach er die placentaren Verkalkungen. Drei Jahre später äußerte er sich über «Die Lösung der mütterlichen Eihäute» (1875); dabei kam er unter einer jetzt nicht mehr gebräuchlichen Nomenklatur zu der noch gültigen Auffassung der Lösung zwischen Zona compacta, die er Großzellenschicht nannte, und Zona spongiosa uteri. Aufgrund der Gefäßanordnung im Endometrium gravidum teilte Langhans, analog wie Reichert (1873), dieses in einzelne Felder ein, woraus er eine Vorstellung über den Blutkreislauf in der Uterusschleimhaut entwickelte. Auf dieser Basis nahm er an, «der Blutstrom, durch die Windungen der Arterien wesentlich geschwächt, würde zunächst dem oberflächlichen Capillarsystem zugeführt werden, und von hier seinen Rückweg in die abführenden Gefäße entweder direkt oder vermittels der eingeschalteten intermediären Venen finden». Dies dürfte die erste wissenschaftliche Arbeit sein, die sich mit der Blutversorgung der Mucosa uteri befaßte. In seiner Publikation «Untersuchungen über die menschliche Placenta» (1877) faßte er seine bisherigen Erfahrungen über das bearbeitete Organ zusammen. Sehr eingehend beschäftigte Langhans und seine Schüler auch die Frage des placentaren Fibrins, so daß wir 
heute aufgrund der entsprechenden Untersuchungen den hypo- oder subchorialen Fibrinstreifen (Langhans) und den oberen Fibrinstreifen (Rohr 1899) als normale physiologische Ablagerungen kennen.

Insgesamt legen 9 eigene und 17 Schülerarbeiten Zeugnis ab vom Einsatz, den Langhans während 40 Jahren der Placenta und anderen embryologischen Problemen widmete (Strauss 1957). Die zweifellos weltweit bekannteste Langhans-Schülerin ist Raissa Nitabuch; sie beschrieb in ihrer Dissertation mit dem Titel «Beiträge zur Kenntnis der menschlichen Placenta» (1887) im Rahmen der Gliederung der Decidua den später nach ihr benannten Nitabuchschen Fibrinstreifen (= basaler Fibrinstreifen). In ihrer Arbeit wies die Doktorandin auf einen in der Nähe der decidualen Oberfläche verlaufenden dunklen Streifen hin, der «die Serotina in zwei Schichten: eine untere, hellere, weniger gefärbte, und eine obere, stärker gefärbte, dunklere» trennt. Den die beiden Schichten trennenden Fibrinstreifen sah Nitabuch als «kanalisiertes Fibrin» an. Die obere Schicht interpretierte sie als Wucherung der «Zellschicht des Chorions» und damit als foetal.

Trotz der damaligen Zeit entsprechenden starken Bindung der embryologischen Arbeiten an die morphologische Forschung sind in allen entwicklungsgeschichtlichen Veröffentlichungen immer wieder Ansätze zur funktionellen Befunddeutung zu erkennen. Somit erweisen sich die embryologischen Publikationen aus dem Langhans-Institut, in dem die entwicklungsgeschichtliche Forschung in Bern nach Valentin ihre große Heimstätte hatte, als ebenso entscheidend wie wertvoll für die Klärung des foetomaternen Stoffwechselorganes. Auf der Basis der vor 100 Jahren in der Bundesstadt geleisteten placentologischen Beiträge konnte erst ein modernes Placentarbild aufgebaut werden.

Sehr ausführlich trat auch Kölliker in seiner Entwicklungsgeschichte (1884) auf die menschlichen Placentarhüllen und die Placenta ein. Die Hüllen trennte er in foetale und mütterliche Hüllen; zu den ersten zählte er Amnion und Allantois und zu den zweiten Chorion und Decidua. Zu den Foetalhüllen wurde meist auch der Dottersack gerechnet, obwohl dieses Gebilde den Embryo nicht umhüllt. Von den Embryonalhüllen sind die Eihüllen zu unterscheiden; darunter sind «alle nicht zelligen Umhüllungen zusammenzufassen», die das unbefruchtete oder befruchtete Ei vom maternen Organismus erhält.

Nach eingehender Beschreibung der verschiedenen Abschnitte der Membrana decidua s. caduca geht Kölliker auf die Zusammensetzung der Placenta ein. Er trennt den Fruchtkuchen in die aus Teilen des Chorions gebildete 
Placenta foetalis vom Mutterkuchen, der Placenta uterina; beide Teile ergeben erst die Gesamtplacenta oder den Mutterkuchen im weitesten Sinn. Den innersten Teil der Placenta uterina nennt er Decidua placentalis und den mächtigeren, nach außen folgenden Abschnitt Pars noncaduca s. fixa placentae uterinae. In der zweiten Schwangerschaftshälfte unterschied Kölliker an der Placenta uterina ein Stratum spongiosum als Homologon der Drüsenlage in der Decidua vera und ein Stratum cellulosum. Nicht unerwähnt darf bleiben, daß er auch Riesenzellen oder vielkernige Zellen in der Decidua placentalis sowie in den Septa fand. Als sehr ungewöhnlich betrachtete Kölliker das Verhalten der Gefässe in der Placenta uterina, weil er sie spiralig sah. Im Anschluß an den Bau und Verlauf der Gefässe trat er auch auf die Zirkulation des mütterlichen Blutes in der Placenta ein. Aufgrund des Gefäßverhaltens müsse der Blutstrom unregelmäßig sein: «Da die Arterien an der konvexen Seite der Placenta zutreten und die Hauptvenen am Rande derselben entspringen, so wird man sagen dürfen, daß der Blutstrom im allgemeinen von der konvexen gegen die konkave Seite und den Rand der Placenta zu geht.» Auf diese Weise sickere das Blut langsam nach den Venentrichtern im konvexen Teil der Placenta hin ab.

Der als Embryologe so bedeutende Anatom Minot (Department of Anatomy, Harvard Medical School, Boston, Mass.) publizierte eine umfangreiche Zusammenfassung diverser Untersuchungseregebnisse zu «Uterus and Embryo» (1889). Dabei ging er von den entsprechenden entwicklungsgeschichtlichen Forschungen am Kaninchen aus, um anschließend die Resultate mit ähnlichen Untersuchungen bei Rodentia zu vergleichen. In Relation zum Meerschweinchen konnte er z. B. feststellen, daß einigen bekannten Voruntersuchern die Metamorphose der Uterindrüsen und der Abbau des foetalen Ektoderms auf der Placenta entgangen war. Die Rattenplacenta hielt er für völlig vergleichbar mit der von Oryctolagus cuniculus. Da Minot fand, die Placenten der verschiedenen Nager seien einander ähnlich, vermutete er, der Placentarbau sei bei allen Rodentiern identisch.

Entschieden ausführlicher geht er dann auf die menschliche Placenta ein und stellt einleitend fest, daß das zweischichtige Chorion aus Mesoderm und Ektoderm besteht. Dieses setzt sich im 1. Schwangerschaftsmonat aus einer äußeren Protoplasma- und einer inneren Zellschicht zusammen; im späteren Graviditätsstadium nehme die ganze Zottenhaut den Charakter einer zellulären Schicht an, die eine Zahl unregelmäßiger Zellflecken über dem Chorion laeve bilde, während die Protoplasmaschicht teilweise oder ganz verschwinde. Im ersten Schwangerschaftsmonat verlieren der Uterus und 
seine Drüsen das Oberflächenepithel, worauf sich die Schleimhaut in zwei Abschnitte teilen läßt; der untere Teil ist cavernös oder schwammig und der obere kompakt, in welchem Bindegewebszellen zu Deciduazellen werden. Im unteren Abschnitt sind die Drüsen in von Zylinderepithel ausgekleidete Areolae verwandelt. Im letzten Schwangerschaftsdrittel ist die Decidua vera von Epithel des ihr anliegenden Chorion laeve bedeckt; auf dem größeren Abschnitt der Decidua serotina liegt Fibrin. Die großen Deciduazellen finden sich nahe dem Chorion, die entsprechenden mehrkernigen Zellen liegen vor allem in der Serotina. Die uterinen Veränderungen während der Menstruation und Schwangerschaft sind homolog, weshalb der mensuelle Zyklus durch die Gravidität verlängert und modifiziert wird. Minot konnte (damals) die Amnionbildung nicht befriedigend erklären. Da die Placenta ein Organ des Chorions ist, kann seine Entwicklung nicht auf eine Modifikation der Allantois oder des Dottersackes zurückgeführt werden. Die Allantois ist schließlich der Darmkanal des Bauchstieles, womit sie die Gefäße zwischen Chorion und Embryo verbindet. Die Allantois vergrößert sich sekundär. Zur Ernährungsfunktion der Placenta sind keine Informationen vorhanden.

Schon bald darauf veröffentlichte Minot eine richtungweisende Arbeit mit dem Titel «Theory of the Structure of the Placenta» (1891), worin er auf die histomorphologische Genese der allanto-chorialen Placenta eingeht. Als Ausgangspunkt dienten ihm die Nachgeburten von Unguiculaten und Ungulaten, deren foeto-maternes Wechselorgan chorial bzw. allantoid genannt werden kann. Die Entwicklung begann bei den Unguiculaten, deren Area placentalis genau am mütterlichen Oberflächenepithel fixiert wird (Beispiel: Hund). Diese choriale Placentation löst anschließend die Auflösung des mütterlichen Epithels und der Drüsen sowie eine Hypertrophe des Stroma endometrii aus, was zur Deciduabildung führt. Dieser unabänderliche Vorgang leite generell die Bildung der Placenta ein, obwohl der genaue Ablauf species-spezifisch differiere. Dadurch ergeben sich verschiedene Verbindungen zwischen foetaler und materner Blutzirkulation. Aus der decidualen chorialen Placenta sei die zonare Placenta der Carnivoren, die diffuse Placenta der niederen Primaten und die metadiscoidale menschliche Placenta abzuleiten. Ausschließlich bei den Unguiculaten ist das Chorion frühzeitig genug für die choriale Placentation dieser Gruppe aufgebaut. Die Huftiere dagegen besitzen keine ausreichende choriale Vaskularisation zur Bildung einer Placenta, weshalb hier die allantoiden Gefäße helfen. Als Resultat dieser Modalität bleibt das mütterliche Blut bei den Ungulaten in 
den maternen Gefäßen, so daß das Chorion durch mütterliches Epithel und Stroma stets von diesen Gefäßen getrennt ist.

Gleichzeitig mit Minot befaßte sich auch H.Strahl (1857-1920) (Prof. der Anatomie in Giessen) (1889) sehr eingehend mit vergleichend-placentologischen Untersuchungen. Sein grundlegendes Verdienst um die Förderung der Placentologie liegt in der von ihm vor 100 Jahren geförderten Anwendung verschiedener Anilinfarben an Schnittpräparaten. So ergaben seine Untersuchungen an Kaninchen, Maulwurf und Hund, daß bei allen drei Tiergruppen vor oder während der festeren Anlagerung des Embryos am Uterus zwecks Bildung der Placenta, es zu einem entweder ganzen oder nahezu vollständigen Verschluß der Uterindrüsen kommt, jedoch ist dieser Vorgang bei den verschiedenen Tieren uneinheitlich.

Abgesehen von seiner primären Histochemie mittels der Anilinfarben hat Strahl mit seinen zahlreichen Publikationen über die Placenta der verschiedenen Säugerspecies wesentliche Beiträge zur vergleichenden Placentologie geleistet. Diese Basis ermöglichte ihm, in seinem Beitrag «Die Embryonalhüllen der Säuger und die Placenta» (1906) eine umfassende comparative Abhandlung zu veröffentlichen. Darin gab er einleitend eine Übersicht über den Aufbau der Embryonalhüllen und schloß daran die Schilderung, wie sich während der intrauterinen Entwicklung der Frucht das amniogene Chorion mit der uterinen Innenwand verbindet. Die bei den Sauropsiden in dieser Entwicklungsphase fast völlig übereinstimmende Bildung und Aufbau der foetalen Hüllen fand Strahl bei den Mammaliern nicht; er stellte vielmehr mancherlei Variationen fest. Bei der Schilderung des Placentarbaues wich er auch von dem von den Systematikern geübten Weg der Säugergruppierung deshalb ab, weil sowohl die Eihüllen als besonders auch der Bau der Placenta «keine vom Einfacheren zum Vollendeteren ... schreitende Reihe zeigt». So begann er auch nicht, der Säugerordnung entsprechend, bei den Monotremen und Marsupialiern, um von hier aufsteigend beim Menschen abzuschlieBen. Bei den Embryonalhüllen der oviparen Säuger erkannte Strahl mit Hubrecht (1889) und van Beneden, daß die Sauropsiden in der Entwicklung der Eihäute keine primitiven, sondern stark modifizierte Verhältnisse aufweisen. Die Entwicklung des Säugeramnions verglich er wiederum unter Berufung auf Hubrecht und van Beneden sowie unter Berücksichtigung der Differenzen mit der Amniogenese bei den Vögeln. Bau und Entwicklung der Säuger-Allantois stimme mehr mit den entsprechenden Verhältnissen bei den Sauropsiden als mit denen bei den Reptilien überein. Bei vergleichender Betrachtung des Placentarbaues kam Strahl zum Schluß, daß bei gleicher 
physiologischer Aufgabe der Aufbau des foeto-maternen Stoffwechselorganes trotz gewisser Grundzüge bei den verschiedenen Tierformen Abweichungen aufweist, die sonst bei Organen gleicher physiologischer Wertigkeit nicht auftreten. Aufgrund dieser Erkenntnis sind nicht die Tierordnungen, sondern nur die verschiedenen Placentarformen in Gruppen nebeneinanderzustellen. So differenzierte er zwischen Mammalia achoria (= Aplacentalia) und Mammalia choriata (= Placentalia); diese trennte Strahl weiter in Halbund Vollplacentalia, denen er weitere Unterordnungen zuschrieb. Für Bau und Entwicklung der menschlichen Placenta kam er schon einleitend zum Schluß, daß hiefür noch das Fundament mit den ersten Entwicklungserscheinungen fehle. Als entscheidendes Zentrum des menschlichen Mutterkuchens sah Strahl mit der überwiegenden Mehrzahl der Autoren den intervillösen Raum, der ein aus einzelnen Abteilungen sich aufbauender Blutsinus sei. In diesem Zentralraum zirkuliere mütterliches Blut, in das die Zotten eintauchen. Duval (1892), Hubrecht und van Beneden hielten den Sinus mit Strahl für aus foetalem Gewebe aufgebaut, während Waldeyer (1826-1921, Anatom in Berlin) (1890) der Ansicht war, der intervillöse Raum bestehe aus erweiterten mütterlichen Gefäßen.

Sehr bald nach Minot und Strahl befaßt sich O.Grosser (1875-1951) zuerst von Wien und dann von Prag aus mit placentologischen Problemen. In zwei bedeutenden Publikationen (1909a, 1927) legte er sehr detailliert seine Interpretationen des unterschiedlichen Baues des Säuger-Mutterkuchens und dessen Funktionen nieder. Wir verdanken ihm eine morphologisch wie systematisch klare Einteilung der möglichen Placentarformen, die im Prinzip noch heute zum Grundstock der Placentologie gehören. In seiner ersten sich auf die vergleichende Placentologie beziehenden Veröffentlichung behandelt Grosser ausführlich die differenten Placentarformen. Hier tritt er wie in der zweiten und entsprechend erweiterten Arbeit (1927) zunächst auf die beiden Formen der Semiplacenta ein und unterscheidet eine Semiplacenta diffusa (Schwein) und Semiplacenta multiplex (Wiederkäuer), die als Placentae appositae zusammengefaßt wurden. An zweiter Stelle folgen die Placentae verae (conjugatae) mit ihren Untertypen Placenta zonaria (meiste Carnivoren) und Placenta discoidalis, die er den Rodentia, Insectivora, Chiroptera und Primates zuordnet. Sehr eingehend wird anschließend die menschliche Placentation besprochen.

Im Anschluß an diese Ausführungen reihte Grosser unter Betonung, daß sich die erwähnten Placentartypen nicht ganz scharf trennen lassen und deshalb Übergangsformen vorkommen, den Versuch an, den Weg der 
Nährstoffe aus dem mütterlichen Gefäßsystem in die Choriongefässe zu verfolgen. Als Versuchsergebnis resultierte die (seither praktisch) akzeptierte Nomenklatur der Placenten in ihrem Verhältnis von Chorion- zu Uterusepithel. So gehen die Termini Placenta epitheliochorialis, Placenta syndesmochorialis, Placenta endotheliochorialis (= Placenta angiotheliochorialis) und Placenta haemochorialis, die in Placenta labyrinthica und olliformis zu gliedern ist, auf Grosser zurück. Die verschiedenen Placentarformen ließen sich nach des Autors Vorstellungen so leicht win eine fortlaufende aufsteigende Reihe ordnen». Weiter vermutete er, daß aufgrund der Homologie der Keimblätter und der foetalen Anhangsorgane eine engere Zusammengehörigkeit der Sautopsiden und der Säuger anzunehmen sei.

Auf der Basis dieser histomorphologischen Aufstellung zog Grosser (1909b) eine Reihe wichtiger Folgerungen. Dabei kam er zum Schluß, daß Embryotrophe und Haemotrophe wesentlich verschieden seien. Aus der Aufstellung schloß er, daß am Unterende der Reihe das Schwein «ausschließlich die embryotrophische Ernährungsform aufweist, während die Wiederkäuer, Raubtiere und Nagetiere so angeschlossen werden könnten, daß von Glied zu Glied der Reihe ein sukzessives Zurücktreten der placentaren Embryotrophe zu konstatieren ist; am anderen Ende der Reihe steht der Mensch mit ausschließlich haemotrophischer Placentarernährung. ... Nur die menschliche Placenta haemochorialis olliformis mit sekundärem, nicht resorbierendem Chorion laeve stellt wieder einen reinen Typus der Ernährung dar, und die bei anderen Species gewonnenen Resultate sind nicht ohne weiteres auf den Menschen übertragbar.»

Zu Ende des 19. und zu Beginn des 20. Jahrhunderts stand auch die Embryologie in ihren vergleichenden Betrachtungen unter dem Einfluß der damals dominierenden anthropozentrischen Einstellung; sie sah im Menschen die Krone der Lebewesen. So kann es nicht überraschen, daß auch die für die vergleichende Placentologie wertvollen Arbeiten von Grosser (1909a u. b, 1925, 1927, 1929 a u. b, 1933, 1936, 1941, 1942) diesen Stempeldruck zeigen. Das damals neue histomorphologische Placentarschema wollte gleichzeitig eine physiologische und phylogenetische Stufenleiter in dem Sinn erkennen lassen, daß die menschliche haemochoriale Placenta der in jeder Weise höchstentwickelte Mutterkuchen sei, während dem gegenüber die Schweineplacenta als epitheliochoriale Halbplacenta an die Basis der foeto-maternen Stoffwechselorgane zu stellen sei.

Sowohl in den jüngeren als auch in den älteren Placentarstadien spielt Fibrin eine wesentliche Rolle, das durch die entsprechenden Untersuchun- 
gen im Langhans-Institut bekannt wurde. Dazu kommen das kanalisierte Fibrin, die Fibrinknoten und die weißen Infarkte. Nach Grossers Auffassung (1925) würden mit dieser Erwähnung verschiedene Dinge zusammengeworfen, weshalb «nur der aus Blut, Lymphe oder Gewebsflüssigkeit niedergeschlagene Faserstoff» Fibrin genannt werden sollte. Diesem Fibrin stellte er eine Reihe geweblicher Abbauprodukte gegenüber, die histologisch dem Fibrin ähneln, aber ihrer Herkunft wegen Fibrinoid zu nennen seien. Die erste Hauptquelle war hier für ihn der Trophoblast, während an zweiter Stelle die Fibrinoidbildung aus zerfallendem mütterlichem Gewebe erfolge.

\section{Schlußbemerkung}

Wenige Jahre vor dem Zweiten Weltkrieg und besonders nach seinem Ende hat die Placentologie-Forschung der verbesserten histologischen, biomolekularen und elektronenmikroskopischen Forschung wegen gewaltige Fortschritte gemacht. Die dadurch erzielten morphologischen Ergebnisse werden heute fortlaufend durch entsprechende physiologische Resultate ergänzt.

\section{Literaturverzeichnis}

Albinus, B.S.: Tabulae septem uteri mulieris gravidae cum jam parturinae mortuas. Leiden: 1748.

Arey, L. B.: Developmental Anatomy. Philadelphia, London: W. B.Saunders Comp., 6th ed., 1959.

Baer, C. E. von: De ovi mammalium et hominis genesi. Leipzig: L. Voss, 1827.

- Untersuchungen über die Gefäßverbindungen zwischen Mutter und Frucht. (Glückwunsch zur Jubelfeier von S. T. Soemmerring) Leipzig: L. Voss, 1828.

Bartelmez, F.W.: Histological studies on the menstruating mucous membrane of the human uterus. Carnegie Inst. Wash., Pub. No. 443; Contrib. Embryol. 142: 141-186 (1933).

Beneden, E. van: La maturation de l'œuf, la fécondation et les premières phases du développement embryonaire des mammifères. Bull.Acad.roy.Soc.Belg. 40: 686-736 (1875).

Bischoff, Th. L. W.: Beiträge zur Lehre von dem menschlichen Foetus. Bonn: 1834.

- Entwicklungsgeschichte der Säugethiere und des Menschen. Leipzig: L. Voss, 1842.

Bloch, B.: Die geschichtlichen Grundlagen der Embryologie bis auf Harvey. Nova Acta Leop.Carol. Dtsch. Akad. Naturforscher 83: 217-334 (1904).

Blumenbach, J.W.: Über den Bildungstrieb (nisus formativus) und seinen Einfluß auf die Generation und Reproduction. Göttingen: Dieterich, 1781. 
Böhme, M.: Vergleichende histologische Untersuchungen über die Uterindrüsen einiger Säuger. Med.-vet. Diss. Bern, 1909.

Bontekoe, K.: Korte Verhandeling van s'Menschen Leven, Gesondheit, Siekte en Dood. Haag: 1685.

Breschet, G.: Etudes anatomiques, physiologiques de l'œuf dans l'espèce humaine et dans quelques-unes des principales familles des animaux vertébrés, pour servir de matériaux à l'histoire générale de l'embryon et du foetus, ainsi qu'à celle des monstruosités ou déviations organiques. Paris: J. B. Baillière, 1833.

Burdach, K. F.: Die Physiologie der Erfahrungswissenschaft. 2. Aufl., Leipzig: 1835.

Cuvier, G.: Mémoire sur les œufs des quadrupèdes. Mém. Mus. d'Hist. Nat., Paris: 3: 82-97 (1817).

De Witt, F.: An historical study on theories of the placenta to 1900. J. History Med. All. Sciences 14: 360-374 (1959).

Duval, M.: Le placenta des rangeurs. Paris: F. Alcan, 1892.

Ercolani, G.B.: Delle glandole otricolari dell'utero e dell'organo glandulare di nuova formazione. Mem. Acad. Sci. Ist. Bologna. 4to. Ser. 2: 133-207 (1868).

Faller, A.: Von Vorstellungen über den Bau der Muskeln bei Galen und den mittelalterlichen Galenisten. Gesnerus 17: 1-13 (1960).

Fischer-Homburger, E.: Krankheit Frau. Bern, Stuttgart, Wien: H. Huber, 1979.

Freind, J.: Emenologia in qua fluxus muliebris menstrui phaenomena, periodi, vita cum medendi methodo, ad rationes mechanicas exeguntur. Oxford: Bennet, 1803.

Goltz, D.: Der leere Uterus. Med.-histor. J. 21: 242-268 (1986).

Grosser, O.: Vergleichende Anatomie und Entwicklungsgeschichte der Eihäute und der Placenta. Wien, Leipzig: W. Braumüller, 1909 a.

- Die Wege der fetalen Ernährung. Slg. anat. physiol. Vorträge Aufs. H. 3: 79-95 (1909b).

- Über Fibrin und Fibrinoid in der Placenta. Z. Anat. Entwickl.-Gesch. 76: 304-314 (1925).

- Frühentwicklung, Einhautbildung und Placentation des Menschen und der Säugetiere. München: J.F.Bergmann, 1927.

- Zur Phylogenese der Placenta. Anat. Anz. 68: 225-304 (1929 a).

- Über die Bedeutung des intervillösen Raumes. Arch. Gynäk. 137: 681-689 (1929b).

- Human and comparative placentation. Lancet: 224: 1-8 (1933).

- Über vergleichende Anatomie und Phylogenese der Placenta. Anat. Anz. 81, Erg.-H. 15-33 (1936).

- Zur Frage der Abstammung der Säugetiere. Erg. Anat. Entwickl.-Gesch. 33: 1-30 (1941).

- Entwicklungsgeschichte des Menschen von der Keimzelle bis zur Ausbildung der äußeren Körperform. Vergleichende und menschliche Placentation. In: L. Seitz und A.I.Amreich, Biologie und Pathologie des Weibes. 2.Aufl., Bd.7: 1-107. Berlin, Wien: Urban und Schwarzenberg, 1942.

Haller, A. von: Anfangsgründe der Physiologie des menschlichen Körpers. Berlin, Leipzig: Ch. F. Voss, Bd. $8 ; 1776$.

Hartman; C.G.: Studies in the reproduction of the monkey Macacus (Pithecus) rhesus, with special reference to menstruation and pregnancy. Carnegie Inst. Wash., Pub. No.433, Contrib. Embryol. 134, 23: 1-191 (1932).

Harvey, W.: Exercitationes de generatione animalium. Quibus accedunt quaedam de partu: de membranis ac humoribus uteri: et de conceptione. Amsterdam: J. Jansson, 1651. 
Herissant, F.D.: Secundinae foetui praestant officis, et sanguine materna foetus non alitur. Paris: 1743.

Hertig, A.T.: Angiogenesis in the early human chorion and in the primary placenta of the macacus rhesus. Carnegie Inst. Wash. Pub. No.501, Contrib. Embryol. No. 145, 25: 37-81 (1935).

Hitschmann, F. und Adler, M.: Der Bau der Uterusschleimhaut des geschlechtsreifen Weibes mit besonderer Berücksichtigung der Menstruation. Mschr. Gebh. Gynäk. 27: 1-81 (1908).

Hoboken, N.: Anatomia secundinae humanae. Utrecht: J. Ribbium, 1669.

Hubrecht, A.A.W.: Studies in mammalian embryology. I. The placentation of Erinaceus europaeus, with remarks on the phylogeny of the placenta. Quart. J. Micr. Sci. 30: 283-404 (1889).

Hunter, J.: The works of John Hunter. Editor: J. F. Palmer; London: Longman, 1835-1837.

Hunter, W.: Anatomia uteri humani gravidi tabula illustrata. Birmingham: J. Baskerville, 1774 .

- An Anatomical Description of the Human Gravid Uterus, and its Contents. London: J. Johnson, 1794.

Hyrtl, J.: Onomotologia anatomica. Geschichte und Kritik der Anatomischen Sprache der Gegenwart. Wien: W. Braumüller, 1880.

- Lehrbuch der Anatomie des Menschen. Wien: W. Braumüller, 1884.

Joerg, J.C.G.: Über das physiologische und pathologische Leben des Weibes. Leipzig: J.C. Heinrichs, 1821.

Kerckring, Th.: Anthropogeniae ichnographia, sive confirmatio foetus ab ovo usque ad ossificationem principiam, in supplementum osteogeniae foetuum. Phil. Trans. 2136-2137 et 2218-2219 (1671).

Kölliker, A.: Grundriß der Entwicklungsgeschichte des Menschen und der höheren Tiere. Leipzig: W. Engelmann, 2. Aufl., 1884.

Langhans, Th.: Zur Kenntnis der menschlichen Placenta. Arch. Gynäk. 1: 317-334 (1870).

- Die Lösung der mütterlichen Eihäute. Arch. Gynäk. 8: 287-297 (1875).

- Untersuchungen über die menschliche Placenta. Arch. Anat. Physiol., Anat. Abt. 188-267 (1877).

- Über die Zellschicht des menschlichen Chorions. Beitr. Anat. Embryol., Festschrift f. Henle 69-79 (1882).

Leopold, G.: Studien über die Uterusschleimhaut während der Menstruation, Schwangerschaft und Wochenbett. Arch. Gynäk. 11 u. 12: 1-146 (1878).

Lobstein, J. F.: Über die Ernährung des Fötus. Halle: Neue Societätsbuchhandlung, 1804.

Meckel, J.F., d. Jüng.: Handbuch der menschlichen Anatomie, Halle, Berlin: Buchhandlg. Hallischen Wissenshauses, Bd.4.(1820).

Minot, Ch.-S.: Uterus and Embryo. J. Morph. 2: 341-458 (1889).

- A Theory of the Structure of the Placenta. Anat. Anz. 6: 125-131 (1891).

Müller-Hess, H.G.: Die Lehre von der Menstruation vom Beginn der Neuzeit bis zur Begründung der Zellenlehre. Abh. Gesch. Med. Naturwiss. H. 27 (1938).

Needham, J.: A History of Embryology. New York: Abelard-Schumann, 2nd. ed., 1959.

Nitabuch, R.: Beiträge zur Kenntnis der menschlichen Placenta. Med. Diss. Bern, 1887.

Oken, L.: Die Zeugung. Hamburg, Würzburg: 1805.

- u. D.G. Kieser: Beiträge zur vergleichenden Zoologie, Zootomie und Physik. Bamberg: 1806. 
Peters, H.: Über die Einbettung des menschlichen Eies und das früheste bisher bekannte menschliche Placentationsstadium. Leipzig, Wien: F. Deutike, 1899.

Plaut, A.: Historical and cultural aspects of the uterus. Ann. N.Y. Acad. Sci. 75: 389-411 (1969).

Ramsey, E. M.: The History of the Uterus. In: H.J.Morris (ed.), The Uterus, 1-16 1973.

- Excitement. In: A.H.Moawand \& M.D. Lindheimer (eds.), Uterine and Placenta Blood Flow, pp. 3-7; Masson Publishing USA Inc., 1982.

Reichert, K.B.: Beschreibung einer frühzeitigen menschlichen Frucht im bläschenförmigen Bildungszustand nebst vergleichenden Untersuchungen über die bläschenförmigen Früchte der Säugethiere und des Menschen. Abh. Kgl. Akad. Wissen., pp. 1-92; Berlin: 1873.

Rohr, K.: Die Beziehungen der mütterlichen Gefässe zu den intervillösen Räumen der reifen Placenta speziell zur Thrombose derselben («weißer Infarkt»). Virchow Arch. 115: 505-534 (1889).

Sabatier, M.: Traité complet d'Anatomie. Paris: 2. Aufl., 1777.

Schröder, R.: Über das Verhalten der Uterusschleimhaut um die Zeit der Menstruation. Mschr. Geburtsh. Gynäk. 39: 3-21 (1914).

- Weibliche Genitalorgane. In: W.v.Möllendorff, Handb. d. Mikrosk. Anat. d. Menschen, Bd.7; Berlin: J. Springer, 1930.

Schuhmann, R.: Placenton: Begriff, Entstehung, Funktionelle Anatomie. In: V.Becker, Th. H.Schiebler, F. Kubli (eds.), Die Placenta des Menschen, pp.199-207. Stuttgart, New York: G. Thieme Verlag, 1981.

Schuhmann, R., R.Borst, G.Geier, H. Kraus: Über die Placentone der reifen menschlichen Placenta. Beih. Z. Geburtsh. Perinat. 1977, pp. 13-26. Stuttgart: F. Enke, 1977.

Soemmerring, S. T.: Icones embryonum humanorum. Frankfurt a. M.: 1799.

Spanner, R.: Mütterlicher und kindlicher Kreislauf der menschlichen Placenta und seine Strombahnen. Z. Anat. 105: 163-242 (1936).

Stieve, H.: Über die Follikelreifung, Gelbkörperbildung und den Zeitpunkt der Befruchtung. Z. mikr.-anat. Forsch. 53: 467-582 (1943).

Strahl, H.: Die Anlagerung des Eies an die Uteruswand. Arch. Anat. Physiol., Anat. Abt. 1889, pp. 213-230 u. Suppl. pp. 197-217.

- Die Embryonalhüllen der Säuger und die Placenta. In: O. Hertwig. Handb. vergl. u. exper. Entwicklungslehre der Wirbeltiere, Bd.1, T. 1, 2. Hälfte, Kap. 8. Jena: G. Fischer, 1906.

Strauss, F.: Theodor Langhans und die Placentologie. Mitt. Naturf. Ges. Bern, N. F. 14: 33-48 (1957).

- Die normale Anatomie der menschlichen Placenta. In: E. Uehlinger (ed.), Hdb. spez. path. Anatomie des Menschen, Bd. VII/5. Berlin, Heidelberg, New York: Springer-Verlag, 1967.

- Das moderne Bild der menschlichen Placenta. Zool. Anz. 208: 289-308 (1982).

- Der weibliche Sexualzyklus. Hdb. Zoologie; Bd.8, Mammalia, Teilbd.55; Berlin, New York: W. de Gruyter, 1986.

Valentin, G.G.: Handbuch der Entwicklungsgeschichte des Menschen. Berlin: A. Rücker, 1835.

Velpeau, A.A.L.W.: Embryologie, ou Ovologie humaine, contenat l'Histoire Descriptive et et Iconographique de l'CEuf Humaine. Paris: J. B. Baillière, 1833.

Waldeyer, W.: Bemerkungen über den Bau der Menschen- und Affenplacenta. Arch. mikr. Anat., 1890. 


\section{Summary}

About 200 years ago, the development of the organ for the fetal-maternal exchange, the placenta, was more or less unknown. The uterus was believed to consist of a number of cavities, especially as a seven-chambered womb. It is very important for structure and completion of the after-birth. Around the beginning of the 16th century Leonardo da Vinci described the human uterus just consisting of one cavity. William Harvey also occupied himself with the uterus. Genesis and problem of menstruation were unknown until the beginning of the 20th century; therefore, the retained menstrual blood was tought to be fetal nourishment. The relationship of ovulation and menstruation also remained unknown on account of insufficient knowledge for a long time. The term "egg", not identical with the germ cell, was used for the content, surrounded by the fetal membranes, of the uterus without the placenta. The germimplantation in utero was an important question for a long time; it was more or less answered during the first half of the 19th century. The term placenta was introduced in the middle of the 16the century. The brothers Hunter started step by step the definition of the different placental parts at that era; with them the science Placentology begins. The problem of bloodcirculation with in the placenta as well as of fetal nourishment was slowly solved at the same time. Placentology improved considerably by comparative investigations during the second half of the 19th century.

Prof. Dr. med. Fritz Strauss

Eichenrain 33

CH-3122 Kehrsatz 